\title{
Cholangioscopic removal of a dislocated stent from the cystic duct using a high pressure biliary dilation balloon
}

A 57-year-old man presented because of painless jaundice. During endoscopic retrograde cholangiopancreatography (ERCP) a papillary neoplasia was diagnosed. Papillotomy was performed and a 10 -Fr transpapillary polyethylene stent was positioned in the common hepatic duct. To avoid dislocation of the stent into the duodenum, additional flaps were cut into the part of the stent located inside the duct.

The patient underwent scheduled ERCP 3 months later for stent exchange; this was done under conscious sedation with propofol. A guide wire was inserted through the stent into the common bile duct. At this point the patient moved on the table, leading to dislocation of the stent into the cystic duct. During an attempt to fasten a Soehendra retriever, the stent further advanced towards the gallbladder. All efforts to grasp the stent with forceps, snare, and Dormia basket were unsuccessful.

We therefore introduced a cholangioscope into the cystic duct and positioned a guide wire under direct endoscopic control through the lumen of the lost stent into the gallbladder ( $\bullet$ Fig. 1 ). The cholangioscope was exchanged for a $5-\mathrm{Fr}$ $4 \mathrm{~cm}$ biliary dilation balloon (Maxforce; Boston Scientific, Natick, Massachusetts, USA), which was positioned over the guide wire inside the stent ( $\bullet$ Fig. 2 ) After the balloon had been inflated, the stent was removed easily.

Endoscopic placement of a stent into the cystic duct has been done for the treatment of cholecystitis [1,2], but complete dislocation of a stent from the hepatic into the cystic duct has not been described in the literature. In our case an unexpected movement of the patient in light sedation caused the loss of the stent into the wrong duct. Extraction with a stone removal balloon placed alongside the prosthesis, as described in the literature [3], was unfeasible due to the small caliber of the cystic duct and the unwanted propulsion of the stent over the spiral folds of the duct (the so-called Heister valves [4]). With the use of a biliary dilation balloon, which is slim enough to be introduced into the lumen of a 10-Fr stent but will provide close contact with the inner wall of the stent over a 4-cm distance when high pressure is applied, firm traction can be applied to the stent. The use of this type of balloon avoids the problems which have been described when a low pressure stone removal balloon (the Fogarty balloon) [5] was used.

Endoscopy_UCTN_Code_TTT_1AR_2AZ
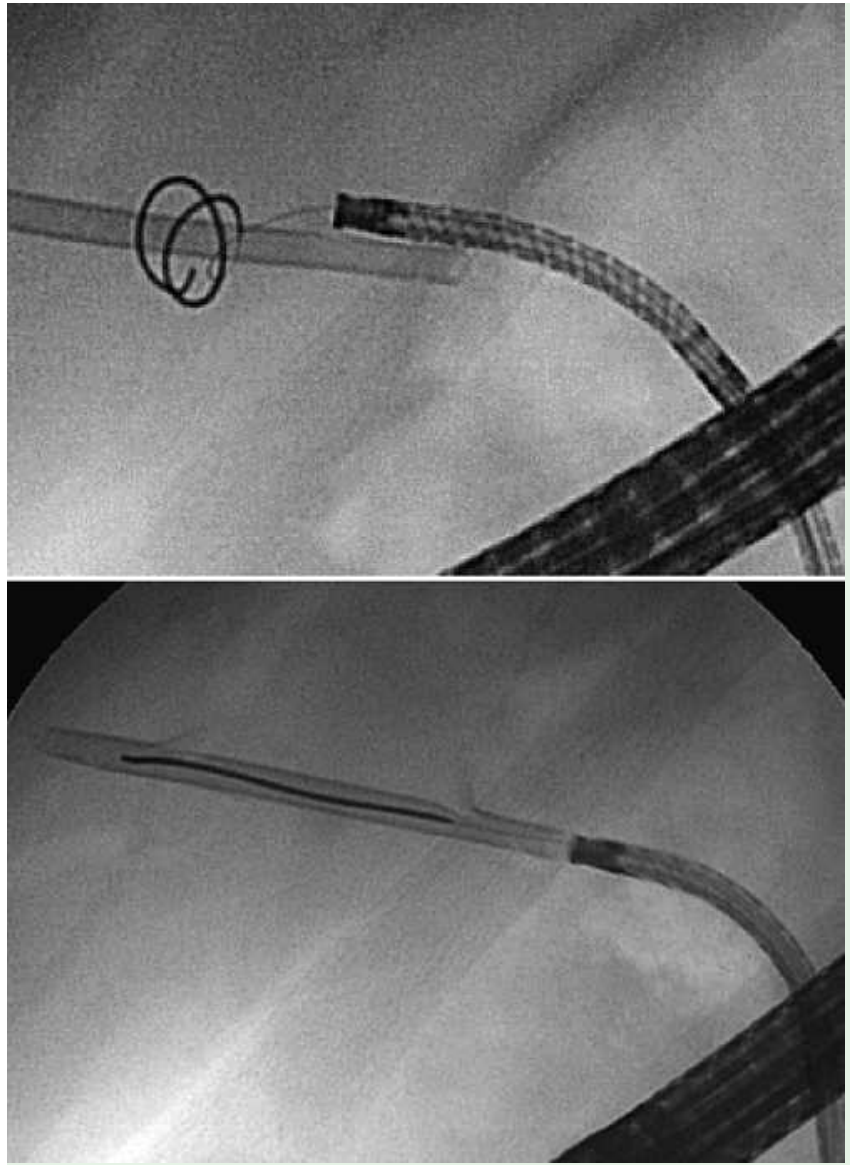

Fig. 1 Upper panel: The cholangioscope is introduced into the cystic duct. The guide wire cannot be advanced alongside the stent because of the Heister valves. Lower panel: Under visual control, the guide wire is successfully introduced into the lumen of the stent. 


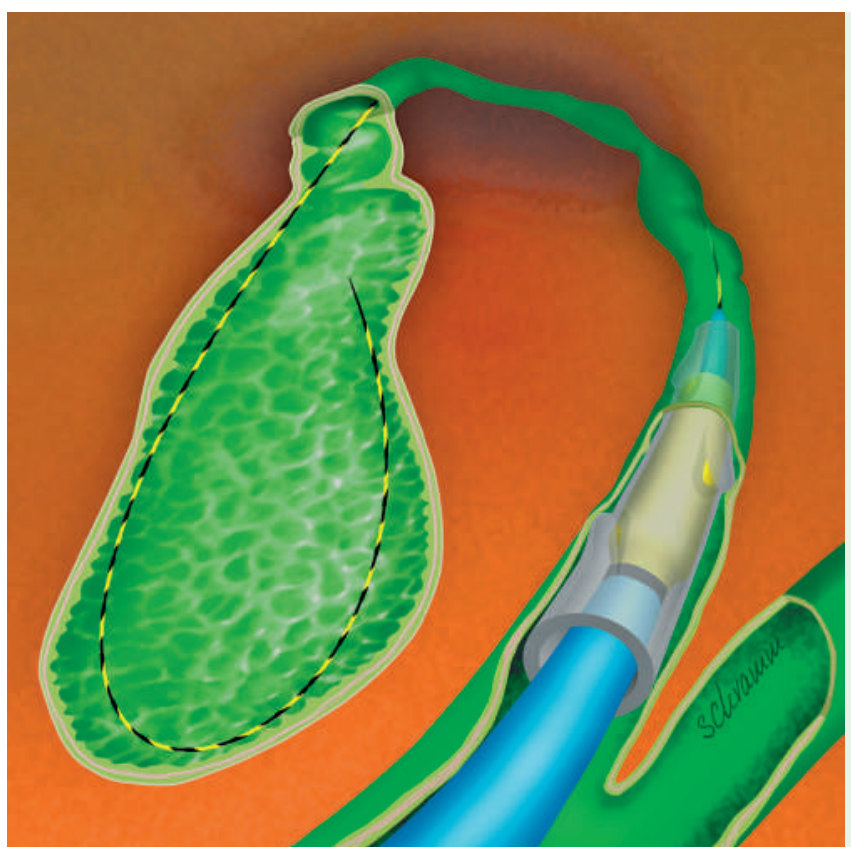

Fig. 2 The guide wire forms a loop inside the gallbladder, allowing easy placement and inflation of a high pressure biliary dilation balloon in the stent lumen.

\section{F. Graepler ${ }^{1}$, A. Raible ${ }^{1}$, H. Neuhaus ${ }^{2}$,} M. Gregor ${ }^{1}$

1 Department of Internal Medicine I, University Clinic Tübingen, Tübingen, Germany

2 Department of Gastroenterology, Evangelisches Krankenhaus Düsseldorf, Düsseldorf, Germany
Bibliography

DOI $10.1055 / \mathrm{s}-2007-966880$

Endoscopy 2007; 39: E337-E338

(c) Georg Thieme Verlag KG Stuttgart · New York . ISSN 0013-726X

\section{Corresponding author}

\section{F. Graepler, MD}

Department of Internal Medicine I

University Clinic Tübingen

Otfried-Müller-Str. 10

72076 Tübingen

Germany

Fax: +49-7071-29-4630

florian.graepler@uni-tuebingen.de 